\title{
ESTÁNDARES PROFESIONALES PARA LA PRÁCTICA PROFESIONAL DEL TRABAJO SOCIAL ESCOLAR EN PUERTO RICO
}




\section{Nancy Viana Vázquez}

Tiene un Doctorado en Educación con especialidad en Administración y Supervisión, y una Maestría en Trabajo Social de la Universidad de Puerto Rico en Río Piedras. Posee un Bachillerato en Artes y cursos adicionales en Educación Especial, Consejería en Rehabilitación y en Literatura de Inglés. Ofrece cursos en el área de Trabajo Social en la Universidad Central de Bayamón, es Facilitadora Docente de Trabajo Social en el Departamento de Educación de Puerto Rico, y ofrece cursos de educación continuada a profesionales del Trabajo Social y otro personal escolar. Ha formado parte de la Facultad Clínica del Departamento de Estudios Graduados de la Universidad de Puerto Rico y pertenece al Consejo Editorial de la Revista Internacional Educación y Aprendizaje. Entre sus publicaciones profesionales, producto de investigaciones se encuentran: Estudio comparativo sobre la deserción escolar en Finlandia y Puerto Rico y Costos y beneficios: La investigación docente en la Universidad de Puerto Rico, Recinto de Río Piedras.

\section{Recibido:}

27 de mayo de 2014

\section{Aprobado:}

1 de septiembre de 2014 


\section{ESTÁNDARES PROFESIONALES PARA LA PRÁCTICA PROFESIONAL DEL TRABAJO SOCIAL ESCOLAR EN PUERTO RICO}

\section{Resumen}

Este artículo presenta los resultados obtenidos en una investigación por encuesta, la cual incluyó la Técnica Delfos y la utilización de cuestionarios para la recopilación de los datos. La muestra incluyó a 114 Trabajadores(as) Sociales Escolares y 15 Facilitadores Docentes de Trabajo Social que laboran en el Departamento de Educación de Puerto Rico (DEPR). Tuvo como propósito identificar los aspectos que deben ser incluidos en los estándares profesionales para trabajadores sociales escolares. Las respuestas ofrecidas por los encuestados evidencian la importancia que le ofrecen los trabajadores sociales escolares a su profesión, ya que entre los aspectos que se identificaron como mayor de nivel de importancia se encuentran los relacionados con aspectos éticos de la profesión, los cuales se presentan a través de las tres categorías 
presentadas en el cuestionario: práctica profesional, desarrollo profesional y estructura administrativa y ética.

Palabras claves: Trabajadores Sociales, Trabajadores Sociales Escolares, Departamento de Educación de Puerto Rico, Colegio de Profesionales del Trabajo Social en Puerto Rico, estándares profesionales, técnica Delfos

\section{Abstract}

This paper presents the results of a survey research, which included the Delphi technique and the use of questionnaires for data collection. The sample included 114 working as Social Facilitators and 15 School of Social Work Teachers working in the Department of Education of Puerto Rico (DEPR). Its purpose was to identify issues that should be included in the professional standards for school social workers. The answers provided by respondents show the importance that school social workers provide to their profession, and that among the issues that were identified as higher level of importance are those related to ethical aspects of the profession, which are presented across the three categories presented in the questionnaire: professional practice, professional development and administrative and ethical structure.

Keywords: Social Workers, Social Workers School, Department of Education of Puerto Rico, School of Social Work Professionals in Puerto Rico, Professional Standards, Delphi Techniques 


\section{Introducción}

La práctica de Trabajo Social Escolar en Puerto Rico se inicia entre las décadas de 1920-1930, con la presencia de las primeras 28 Maestras Visitantes en las Segundas Unidades Rurales del Departamento de Instrucción Pública, tal como se le conocía en aquella época al Departamento de Educación de Puerto Rico (DEPR) (Burgos, 2002). En el año 2012, el DEPR ya contaba con 1,800 Trabajadores Sociales Escolares aproximadamente, ubicados en las escuelas, Distritos Escolares y Regiones Educativas (Rivera, 2010). Este incremento denota la importancia y necesidad que tomó la presencia de estos profesionales en el DEPR. No obstante, según han ido pasando los años, incrementan los retos para la profesión.

En este artículo se presentan los resultados de una investigación llevada a cabo por esta investigadora el año escolar 2012-2013, por encomienda de la Directora del Programa de Trabajo Social en ese entonces, Inés Rivera Colón. Esta investigación tuvo como propósito identificar parte del contenido que debía incluir la actualización de los estándares para la práctica de trabajo social escolar en Puerto Rico, de acuerdo a las normativas vigentes. Como preguntas de investigación se establecieron las siguientes: ¿Qué aspectos deben incluirse en la actualización de los Estándares Profesionales para la Práctica del Trabajo Social Escolar en Puerto Rico? ¿Cuáles de estos presentan mayor nivel de importancia?

\section{Marco conceptual}

El marco conceptual de esta investigación se nutrió de la revisión de las diferentes normativas relacionadas con lo que es la práctica profesional de trabajo social, el trabajo social escolar, tanto en Puerto Rico, como en otras partes del mundo, 
como por ejemplo, Estados Unidos, Canadá y Australia. En cuanto a las normativas que rigen a los(as) Trabajadores(as) Sociales Escolares (TSE) en Puerto Rico, además de la Ley Núm. 171 de 1940, Ley de Colegiación de Trabajo Social en Puerto Rico según enmendada, se les requiere, como área de especialidad de la profesión, incluir conocimientos adicionales para ser certificados como Maestros en Trabajo Social Escolar (Reglamento del Personal Docente de Puerto Rico, 2004).

Por la complejidad de las necesidades que son atendidas en el escenario escolar, cada año escolar surgen nuevas normativas o son actualizadas las existentes $y$, aunque en este artículo no se mencionarán todas las relacionadas con el Programa de Trabajo Social Escolar, se incluyen algunas de las más utilizadas y conocidas, tales como: Carta Circular-3-2010-2011 (2010) Normas para la Organización y Funcionamiento del Programa de Trabajo Social Escolar, Manual de Procedimientos de Trabajo Social (2012), Manual de Procedimientos del Programa de Educación Especial (1988), Manual de Protocolos: Guía para el Director Escolar y Personal de Apoyo (2011), entre otros. Lo antes expuesto justifica la revisión y actualización de los Estándares Profesionales de Trabajo Social Escolar.

Según la Asociación Nacional de Trabajadores Sociales (2012), los estándares profesionales permiten a los trabajadores (as) sociales escolares estar alerta sobre las destrezas, conocimientos, valores, métodos y sensibilidad necesarios para trabajar efectivamente en los sistemas escolares. Las competencias se definen como la síntesis de la conducta profesional que integra el conocimiento, las destrezas y actividades en la ejecución de las tareas del trabajador social escolar. De acuerdo a la Carta Circular 3-2010-2011 Normas y funcionamiento del Programa de Trabajo Social Escolar, el TSE se define como el profesional de la conducta humana en la 
comunidad escolar, que se especializa en fortalecer y fomentar el máximo funcionamiento social de los estudiantes, integrando los diferentes grupos que interactúan con este: personal escolar, familia y comunidad (Departamento de Educación, 2010).

Diferentes lugares del mundo cuentan con estándares profesionales para TSE. Por ejemplo, Australia cuenta con estándares para estos profesionales, que se organizan en las siguientes áreas principales: Práctica Directa, Servicios Administrativos, Desarrollo Organizacional y Sistema de cambio, Política, Investigación y Desarrollo Profesional (Asociación Australiana de Trabajadores(as) Escolares, 2003). Así mismo, algunos estados de los Estados Unidos cuentan con estándares que se atemperan a sus necesidades particulares, de acuerdo a las normativas vigentes en la práctica profesional. Por ejemplo, en los estados de Connecticut (2014) y de Carolina del Norte (Public Schools of North Carolina, 2008) cuentan con estándares específicos para la práctica de esta especialidad.

A pesar de que no se encontraron estándares profesionales categóricamente diseñados para trabajadores sociales escolares en otros países de Latinoamérica, sí se encontró un documento generado por la Red Latinoamericana de Acogimiento Familiar, UNICEF (2010). El documento, "Guía de Estándares para el personal de las entidades públicas y privadas que se ocupan de la protección de derechos de niñas, niños y adolescentes", incluye los estándares, principios y orientaciones generales: prevenir la separación, cuándo se decide la separación, el cuidado alternativo, promover la reintegración a la familia y/o dar soluciones estables y el cuidado alternativo en situaciones especiales. Establecen que este documento, en su implementación fundamental, va dirigido a promover la garantía del derecho a la convivencia familiar y comunitaria de los niños, las niñas y los adolescentes latinoamericanos. 


\section{Metodología}

Se utilizó una metodología de corte cuantitativo, cuyo diseño fue una investigación por encuesta. Fowler (2002) indica que las encuestas tienen como propósito producir estadísticas. También plantea que la forma de recopilar los datos se lleva a cabo realizando preguntas, y que generalmente se recopila a una fracción de la población que se desea estudiar.

Para la encuesta se utilizaron dos cuestionarios como instrumentos para recopilar los datos. Según Fowler (2002), existen algunas ventajas al utilizar los cuestionarios. El costo económico y en términos de tiempo es menor, se puede abarcar en menos tiempo a mayor cantidad de personas, y hay menor riesgo de que se influya directamente sobre las respuestas de los encuestados, entre otras.

La recolección de datos se llevó a cabo en dos etapas, utilizando la Técnica Delfos o de juicio de expertos (Linstone \& Turrof, 2002). En la primera etapa, luego que el instrumento fuera evaluado por 3 expertos, se le administró a 15 Facilitadores Docentes de Trabajo Social (FDTS). En esta primera ronda, las instrucciones fueron que escogieran todos los ítems que entendían debían incluirse como parte de los estándares profesionales. También podían realizar recomendaciones en la redacción de las aseveraciones y añadir cualquier otro dato que no se hubiese incluido y que consideraran necesario. En la segunda etapa, se administró el cuestionario ya con las últimas correcciones recomendadas. En esta segunda etapa, además de los Facilitadores Docentes de Trabajo Social (FDTS), también se incluyó a trabajadores sociales escolares. 


\section{Muestra}

La muestra que participó de esta investigación fue una no probabilística y por conveniencia, ya que la administración de los cuestionarios a los trabajadores sociales escolares se llevó a cabo en un Taller Residencial del Programa de Trabajo Social Escolar, efectuado durante el mes de junio, en el municipio de Río Grande, por acuerdos previos con la Directora del Programa. Según Verhoeven (2011), el tipo de muestra no probabilístico se utiliza cuando no es posible seleccionar la muestra de manera aleatoria. No obstante, la participación de estos fue voluntaria y anónima, para así proteger la privacidad de los participantes.

Los encuestados provenían de diferentes partes de Puerto Rico, ya que el Taller Residencial del Programa de Trabajo Social Escolar tenía como criterio que la participación fuera representativa de los 28 distritos escolares. Finalmente la muestra total fue de 114 TSE y de los 15 FDTS.

\section{Instrumento}

El instrumento utilizado para recopilar los datos consistió de un cuestionario que fue desarrollado luego de realizar la revisión de literatura, tanto de las normativas relacionadas con la profesión de Trabajo Social Escolar en Puerto Rico, así como en otras partes del mundo. Las categorías incluidas en las que se organizaron los ítems fueron las siguientes: práctica profesional, preparación y desarrollo profesional, y estructura administrativa. Una vez desarrollado el cuestionario, se presentó para el juicio de 3 expertos según recomienda Verhoeven (2011). Estos evaluaron la construcción de los ítems, la clasificación y si se debía añadir algún otro. En el juicio 
de expertos, en lo que se refiere al análisis de las preguntas, se le prestó especial atención al contenido. En cuanto a los expertos en diseño, se analizaron las preguntas para evaluar si las preguntas cumplían con los estándares de calidad: contenido, cognitivo y de utilidad (Fowler, 2002).

\section{Resultados y análisis}

Los hallazgos están presentados de acuerdo con las categorías incluidas en el cuestionario. El instrumento consistió de preguntas categorizadas o de selección múltiple, en escala de abanico abierto (Ander - Egg, 1980), lo que significa que las respuestas de los participantes fueron contestadas escogiendo entre un grupo de alternativas en cada categoría.

Con el objetivo de facilitar el análisis de los datos, se colocaron los ítems de mayor a menor Nivel de Importancia (NI), de acuerdo el Valor Asignado por los encuestados. Para obtener el $\mathrm{Nl}$, se le otorgó el valor de 1 por cada selección que le realizaran los encuestados ( $N$ ) a ese ítem. Esto se representa en la siguiente ecuación $\sum \mathrm{N}=\mathrm{NI}$. Para la parte del análisis de los resultados mencionaré los primeros cinco ítems de mayor Nivel de Importancia de acuerdo con los(as) encuestados(as). Esto no significa que el resto de los hallazgos no sean importantes, sin embargo, se realizó de esta manera para cumplir con los estándares para la publicación de este artículo. 


\section{Tabla 1. Nivel de importancia de}

los Estándares de Práctica Profesional

\section{Ítem}

1 Establece una relación profesional desde el inicio hasta el final del proceso de intervención y establece los límites de la confidencialidad establecidos por ley.

2 Demuestra compromiso con los valores y ética de la profesión y el Código de Ética del Colegio de Profesionales del Trabajo Social en Puerto Rico.

3 Mantiene las medidas de seguridad necesarias para garantizar la privacidad y confidencialidad de la información obtenida de sus participantes en colaboración con la administración de la escuela.

4 Ofrece a sus estudiantes servicios dirigidos al desarrollo de fortalezas individuales que les permitan maximizar sus oportunidades en la planificación y dirección de sus experiencias de aprendizaje.

5 Trabaja en colaboración con el personal de otras agencias (interagencial) en la movilización de recursos para atender las necesidades de los estudiantes y las familias.

6 Aboga por los derechos de los estudiantes y sus familias en diferentes contextos que inciden en el aprovechamiento escolar.

7 Ofrece y coordina adiestramientos, talleres y proyectos de prevención, a tono con las metas y misión del Departamento de Educación.

8 Involucra al cliente en el desarrollo del plan de intervención, y su implantación incluye las fortalezas y capacidades de este. 109

9 Posee conocimiento de las etapas de desarrollo de los niños y adolescentes, y las aplica en sus intervenciones sociales.

10 Colabora en el apoderamiento del estudiante y sus familias para que puedan tener acceso y utilizar de manera efectiva las redes de apoyos formales e informales. 
11 Conoce los indicadores de maltrato, las políticas escolares, las legislaciones vigentes, los procesos y los protocolos estatales y federales para atender estas situaciones.

12 Ofrece consultoría al personal escolar y miembros de la comunidad de aprendizaje para promover el conocimiento y la utilización efectiva de los servicios de Trabajo Social Escolar. 104

13 Posee las destrezas para realizar trabajos colaborativos y demuestra dominio del trabajo en equipo.

14 Posee conocimiento sobre la dinámica familiar durante los procesos de intervención social.

15 Lleva a cabo procesos de avalúo en la identificación de las necesidades de sus estudiantes de manera individualizada o grupal, lo cual facilita el desarrollo del plan de intervención a utilizarse en la comunidad de aprendizaje.

16 Al trabajar en equipos multidisciplinarios, logra mantener los principios, valores y prácticas de la profesión.

17 La información recopilada durante el ofrecimiento de servicios es guardada y mantenida de manera confidencial.

18 Demuestra estar adiestrado y preparado en el uso de la mediación y estrategias de resolución de conflictos para promover un entorno escolar adecuado en la escuela y comunidad para la adquisición de relaciones saludables.

19 Mantiene datos actualizados y relevantes en la planificación, administración y evaluación de los servicios que ofrece.

20 Posee el conocimiento, las destrezas y recursos necesarios para atender las necesidades de los estudiantes y sus familias. 96

21 Organiza su tiempo y ocupa su energía en el manejo de casos, de manera que pueda cumplir con las responsabilidades y tareas asignadas, estableciendo prioridades.

22 En su rol de agente de cambio, identifica áreas de necesidad que no han sido atendidas por la escuela y la comunidad y puede colaborar en el desarrollo y búsqueda de servicios que cubran estas necesidades. 
23 Está alerta y pendiente de la relación establecida entre profesional y cliente, así como de su contexto social y las acciones a realizarse.

24 Redacta informes que revelan las circunstancias precisas y objetivos del cliente, tomando en cuenta los principios éticos y legislativos establecidos por ley.

25 Se asegura que los estudiantes y sus familias reciban los servicios de acuerdo a su contexto cultural, de manera que posean las competencias para mejorar las experiencias de aprendizaje de sus hijos.

26 Incorpora el proceso de avalúo en el desarrollo e implantación de los planes de intervención social de los estudiantes.

27 El avalúo y la intervención social son apropiados de acuerdo a la situación del cliente y los requisitos éticos y legislativos establecidos.

En la Tabla 1 se presenta las puntuaciones obtenidas de acuerdo con el Nivel de Importancia otorgado a cada ítem por los encuestados en la categoría de Estándares de Práctica Profesional. El ítem que obtuvo mayor Nivel de Importancia con un total de 126 indicaba lo siguiente: establece una relación profesional desde el inicio hasta el final del proceso de intervención y establece los límites de la confidencialidad establecidos por ley. Esto cumple con lo establecido en el Canon de Ética, él y la Profesional de Trabajo Social, y los (as) participantes y el Canon III, El Profesional de Trabajo Social y la Confidencialidad (Código de Ética Profesional, 2011).

El ítem que ocupó el segundo lugar Nivel de Importancia con 123 puntos de indicaba: demuestra compromiso con los valores y ética de la profesión y el Código de Ética del Colegio de Profesionales del Trabajo Social en Puerto Rico 
(2011). La Asociación Nacional de Trabajadores Sociales, en su documento de Estándares para los Servicios de Trabajo Social escolar (2012), establece en su Estándar Núm. 1 Ética y Valores que los TSE deben demostrar valores tales como: el servicio, justicia social, integridad, entre otros, adheridos a su práctica profesional.

El tercer ítem en obtener el mayor Nivel de Importancia con 119 puntos, indicaba lo siguiente: mantiene las medidas de seguridad necesarias para garantizar la privacidad y confidencialidad de la información obtenida de sus participantes en colaboración con la administración de la escuela. Este ítem incluye tanto los documentos físicos como los virtuales, de acuerdo al Código de Ética Profesional (2011). Además, guarda relación con lo dispuesto en el Estándar 1 de la Asociación de Trabajadores Sociales de Saskatchewan en Canadá y su documento, Estándares para Trabajadores Sociales Escolares Registrados (1999). Así también se relaciona con el Estándar 5, el cual estipula que los trabajadores sociales deben mantener la confidencialidad de manera que promueva el mejor bienestar de su cliente.

El cuarto ítem, con un total de 115 puntos de Valor Asignado, indicaba: ofrece a sus estudiantes servicios dirigidos al desarrollo de fortalezas individuales que les permitan maximizar sus oportunidades en la planificación y dirección de sus experiencias de aprendizaje. Por último, el ítem en la quinta posición, con 114 puntos, decía lo siguiente: trabaja en colaboración con el personal de otras agencias (interagencial) en la movilización de recursos para atender las necesidades de los estudiantes y las familias. Estos dos últimos ítems guardan relación directa con la política pública establecida por el Departamento de Educación y su CC 9-2010-2011 Normas de Organización y Funcionamiento del Programa de 
Trabajo Social Escolar (2010). De igual modo, para los TSE de Australia, de acuerdo a sus Estándares para la Práctica para Trabajadores(as) Sociales Escolares (2008), se requiere que estos tengan el conocimiento, las destrezas y recursos necesarios para atender la situación del/la cliente, en este caso del estudiante y sus familias.

\section{Tabla 2. Nivel de importancia de los Estándares de Preparación Académica y Desarrollo Profesional}

\begin{tabular}{llc} 
& Ítem & NI \\
\hline 1 & $\begin{array}{l}\text { Posee los conocimientos básicos de la profesión de } \\
\text { Trabajo Social aplicados al entorno escolar. }\end{array}$ & 104 \\
\hline 2 & $\begin{array}{l}\text { Asume la responsabilidad y cumple con su propio desarrollo } \\
\text { profesional de acuerdo a lo establecido por el Colegio }\end{array}$ & \\
& de Profesionales del Trabajo Social en Puerto Rico. & 98 \\
\hline 3 & $\begin{array}{l}\text { Toma las previsiones necesarias para cumplir con las prácticas } \\
\text { establecidas por el Colegio de Profesionales del Trabajo }\end{array}$ & 94 \\
\hline & $\begin{array}{l}\text { Social en Puerto Rico. } \\
\text { Conoce y entiende en términos generales la organización y }\end{array}$ & \\
& $\begin{array}{l}\text { funcionamiento del Departamento de Educación en sus } \\
\text { diferentes niveles. }\end{array}$ & 90 \\
\hline
\end{tabular}

5 Posee conocimiento y comprende el efecto que pueden tener sobre el estudiante las relaciones recíprocas del hogar, la escuela y la comunidad.

6 Entiende y trabaja con los entornos de los que provienen los estudiantes y cuenta con una amplia variedad de experiencias que ayudan en la formación integral del estudiante y su aprendizaje.

7 Posee el conocimiento y la disponibilidad para la selección y aplicación de metodologías de intervención en prácticas basadas en evidencia (PBE), tanto en prevención como en intervención individual. 
8 Posee las destrezas de evaluar su práctica profesional y divulga los hallazgos a la comunidad de aprendizaje y a su profesión.

9 Posee las actitudes y destrezas para promover la colaboración entre los proveedores de servicios de salud física y mental, sus estudiantes y familias.

10 Entiende las relaciones entre la práctica y las políticas que afectan al estudiante.

11 Contribuye al desarrollo profesional de otros al recibir, educar y supervisar practicantes de trabajo social en su centro de trabajo. 69

12 Posee las destrezas de avalúo sistemático y de investigación social que aplican en los procesos de intervención.

13 Posee destrezas en el desarrollo de coaliciones y/o alianzas a nivel local, estatal y nacional que promueven el éxito estudiantil. 43

En la Tabla 2 se presenta la categoría de los Estándares de Preparación Académica y Desarrollo Profesional. El ítem de mayor Nivel de Importancia, con un total de 104, indicaba: posee los conocimientos básicos de la profesión aplicados al entorno escolar. En segundo lugar, con 98 puntos, el ítem decía: asume la responsabilidad y cumple con su propio desarrollo profesional de acuerdo a lo establecido por el Colegio de Profesionales del Trabajo Social en Puerto Rico y los requisitos del estado. En tercer lugar, con 94, el ítem decía lo siguiente: toma las previsiones necesarias para cumplir con las prácticas establecidas por el Colegio de Profesionales del Trabajo Social en Puerto Rico. En los tres ítems, resulta necesario que cada TSE cuente con la preparación académica necesaria para ejercer de manera adecuada la práctica profesional, lo que también concurre por lo dispuesto por la Ley Núm. 171 de 1940 según enmendada, de Colegiación de Trabajo Social en Puerto Rico. 
En cuarto lugar quedaron dos ítems, los cuales obtuvieron una puntuación similar de 90 puntos. El primero indicaba: conoce y entiende en términos generales la organización y funcionamiento del Departamento de Educación en sus diferentes niveles. El segundo ítem decía: posee conocimiento y comprende el efecto que pueden tener las relaciones recíprocas del hogar, la escuela y la comunidad en el estudiante.

En quinto lugar, con 79, quedó el ítem que indicaba: entiende y trabaja con los entornos de los cuales provienen sus estudiantes, y cuenta con una amplia variedad de experiencias que ayudan en la formación integral del estudiante y su aprendizaje.

\section{Tabla 3. Nivel de importancia de los Estándares para la Estructura Administrativa y de Apoyo}

Ítem

NI

1 El Departamento de Educación es la agencia responsable de otorgar las certificaciones correspondientes de Trabajador Social Escolar y Facilitador Docente de Trabajo Social de acuerdo a las credenciales y experiencias del solicitante.

2 El Colegio de Profesionales del Trabajo Social en Puerto Rico regula la práctica del Trabajo Social Escolar en colaboración con el Departamento de Educación.

3 Los trabajadores sociales escolares empleados por el Departamento de Educación cuentan con las credenciales que requieren el Reglamento de Personal Docente y el Departamento de Educación, además de los requeridos por el Estado.

4 El salario y clasificación de los Trabajadores Sociales Escolares está basado en su educación, experiencia y responsabilidades.12

5 El Departamento de Educación provee a los Trabajadores Sociales Escolares el ambiente de trabajo adecuado que permite una práctica efectiva de acuerdo a los recursos disponibles. 
6 Las metas, los objetivos y tareas del Programa de Trabajo Social Escolar se encuentran claramente relacionadas y dirigidas hacia la misión del Departamento de Educación y el proceso educativo. 12

7 Todos los programas del Departamento de Educación que incluyen los servicios de Trabajo Social Escolar son evaluados por sus participantes.

8 El Departamento de Educación empleará a los Trabajadores Sociales Escolares con los niveles más altos de calificaciones, de acuerdo a lo que establece la política del Departamento de Educación.

9 El Departamento de Educación involucra a los Trabajadores Sociales Escolares en el desarrollo y coordinación de acuerdos con la comunidad de salud, salud mental y los proveedores de servicios sociales articulados con las escuelas, para asegurarse de que estos servicios promueven el éxito estudiantil.

10 El Departamento de Educación provee las oportunidades a los Trabajadores Sociales Escolares para participar en actividades de desarrollo profesional dirigidas hacia la misión y visión de agencia y del programa.

11 El Departamento de Educación establece la cantidad o la proporción estudiantil que puede ser atendida por cada Trabajador Social Escolar para asegurar unas expectativas en el manejo de los casos (por ejemplo 1:250).

12 La administración del Departamento de Educación establece los procesos de supervisión y asistencia técnica ofrecida a los Trabajadores Sociales Escolares.

13 Los trabajadores sociales en las escuelas son designados como Trabajadores Sociales Escolares.

La Tabla 3 presenta los ítems relacionados con la Estructura Administrativa y de Apoyo para los TSE. En esta categoría de los Estándares para la Estructura Administrativa y de Apoyo, 
solo pudieron participar 13 de los 15 Facilitadores Docentes de Trabajo Social, ya que tendrá una fase adicional con otros expertos en el área. Con el mayor Nivel de Importancia, se encuentra el ítem con un Nivel de Importancia de 13 puntos. El ítem decía: el Departamento de Educación otorga las certificaciones correspondientes de Trabajador Social Escolar y Facilitador Docente de Trabajo Social, de acuerdo a las credenciales y experiencias del solicitante. De acuerdo al Reglamento de Personal Docente (2004), se requiere que el TSE cuente con una certificación en la categoría de Trabajador Social Escolar y es esta la agencia encargada de otorgar dicha certificación a los (as) trabajadores (as)que cumplen con los requisitos correspondientes.

En segundo lugar, con un Nivel de Importancia de 12 puntos, quedaron los siguientes 6 ítems: el Colegio de Profesionales del Trabajo Social en Puerto Rico regula la práctica del TSE en colaboración con el DEPR; los TSE empleados por el DEPR cuentan con las credenciales que requiere el Reglamento de Personal Docente y el DEPR, además de las requeridos por el Estado; el salario y clasificación de los trabajadores sociales escolares están basados en su educación, experiencia y responsabilidades; el DEPR provee a los TSE el ambiente de trabajo adecuado que permite una práctica efectiva de acuerdo a los recursos disponibles. Otro fue: las metas, los objetivos y tareas del Programa de Trabajo Social Escolar se encuentran claramente relacionadas y dirigidas hacia la misión del DEPR y el proceso educativo; y por último, todos los programas del Departamento de Educación que incluyen los servicios de Trabajo Social Escolar son evaluados por sus participantes.

Los primeros dos ítems: el Colegio de Profesionales del Trabajo Social en Puerto Rico regula la práctica del TSE en colaboración con el DEPR y los TSE empleados por el DEPR cuentan con las credenciales que requiere el Reglamento 
de Personal Docente y el DEPR, se establecen como parte de la Ley Núm. 171 de 1940, según enmendada. El ítem que indicaba: el salario y clasificación de los trabajadores sociales escolares está basado en su educación, experiencia y responsabilidades, forma parte del Reglamento de Carrera Magisterial del Departamento de Educación (1999), en el cual IOs TSE pueden aumentar su salario de acuerdo a su educación y experiencia en la agencia. No obstante, a los FDTS no les aplica este Reglamento, ni a lOs TSE que no cuenten con permanencia en su puesto. Cabe señalar que, a pesar de que no se pudo comparar los salarios básicos de IOs TSE de Puerto Rico con los de otros países, se incluye el salario básico de un Trabajador Social en Puerto Rico, el cual es de $\$ 1,750$. En Estados Unidos, la remuneración promedio anual, para los Trabajadores Sociales Escolares fluctuaba en $\$ 60,480$, lo que equivale a un salario mensual de $\$ 5,040$ (Oficina de Estadísticas Laborales de Estados Unidos, 2013).

El otro ítem que indicaba que el DEPR provee a los TSE el ambiente de trabajo adecuado que permite una práctica efectiva de acuerdo a los recursos disponibles, a pesar de que forma parte de la CC 3-2010-2011, basado en la experiencia de la investigadora como Trabajadora Social Escolar durante 13 años y FDTS durante 3 años, el mismo no se cumple a cabalidad. De acuerdo a la experiencia de la investigadora en el DEPR, muchos TSE no cuentan con muchos de los recursos necesarios para realizar sus funciones, tales como: oficinas accesibles y libres de ruidos, espacios físicos para trabajar terapia de grupo, teléfonos, computadoras, fotocopiadoras, entre otros. Estos datos se basan principalmente en los datos obtenidos de las propias voces de las TSE cuando se llevan a cabo las reuniones mensuales, a través de llamadas telefónicas que se reciben durante el transcurso del año escolar y por las visitas que realiza la FDTS, como parte de sus funciones. 
El otro de los ítems que obtuvo el segundo Nivel de Importancia fue el siguiente: las metas, los objetivos y tareas del Programa de Trabajo Social Escolar se encuentran claramente relacionadas y dirigidas hacia la misión del DEPR y el proceso educativo. Cabe señalar que a pesar de que siempre cuentan con retos para mantenerse actualizados y al día, por el constante cambio y revisión de normativas de la agencia, es importante indicar que falta actualizar ciertos documentos de trabajo, tales como: Ios Planes de Trabajo Anuales, Instrumento de Evaluación del Desempeño del Trabajador Social Escolar y de los Facilitadores Docentes de Trabajo Social, entre otros.

Por último, el ítem indicaba que todos los programas del Departamento de Educación que incluyen los servicios de Trabajo Social Escolar son evaluados por sus participantes. Al momento de redactar este artículo, el Programa de Trabajo Social Escolar no llevaba a cabo procesos de evaluación de sus servicios por parte de sus participantes, con excepción de algunas de las actividades de prevención. Este proceso de evaluación de los servicios de TSE se recomienda que sean evaluados por sus participantes, tanto por la Asociación de Trabajadores Sociales de Saskatchewan (1999), como por la Asociación Nacional de Trabajadores Sociales (2012).

\section{Recomendaciones}

Entre las implicaciones que se incluirán en esta sesión mencionaré las relacionadas con el Departamento de Educación de Puerto Rico para el Colegio de Profesionales del Trabajo Social, las universidades, para los FDTS y TSE. 


\section{Departamento de Educación Programa de Trabajo Social Escolar}

1. Actualización de los Estándares Profesionales de Trabajo Social Escolar, tomando en consideración los resultados de esta investigación.

2. Articular todos los documentos y normativas relacionadas con el Programa a los nuevos Estándares Profesionales.

3. Divulgar y discutir los Estándares con la población escolar, una vez estén listos.

4. Establecer un plan para la revisión continua de los documentos del Programa de manera integral y sistemática.

5. Establecer lazos de cooperación entre el Colegio de Profesionales de Trabajo Social en Puerto Rico y las universidades, entre otras organizaciones.

\section{Colegio de Profesionales del Trabajo Social en Puerto Rico}

1. Establecer lazos de colaboración para trabajar en el proceso de actualización de los Estándares Profesionales de Trabajo Social Escolar y otros asuntos.

2. Divulgar los Estándares Profesionales de Trabajo Social Escolar entre los miembros del Colegio de Profesionales del Trabajo Social.

3. Continuar ofreciendo adiestramientos y talleres especializados para estos profesionales, basado en sus áreas de necesidad.

4. Considerar la posibilidad de constituir una Comisión Permanente de Trabajo Social Escolar, de manera que se puedan estrechar más los lazos de colaboración. 


\section{Universidades- Programas de Trabajo Social}

1. Mantener alianza con el Programa de Trabajo Social Escolar y las escuelas, de manera que puedan mantener sus currículos actualizados de acuerdo con las necesidades emergentes.

2. Colaborar en el proceso de actualización de los Estándares Profesionales de Trabajo Social Escolar.

3. Ofrecer actividades de desarrollo profesional para Trabajadores Sociales Escolares, tales como trabajos de investigación, proyectos especiales, entre otros.

\section{Facilitadores Docentes de Trabajo Social}

1. Mantenerse actualizados y a la vanguardia de todos los cambios en la práctica de TSE y de la profesión en general.

2. Continuar colaborando en la actualización de los Estándares y de otras áreas requeridas del Programa de Trabajo Social Escolar.

3. Mantener a los TSE asignados a su cargo informados de los Estándares y de las normativas vigentes de la profesión.

4. Establecer alianzas con universidades y otras agencias para los planes de desarrollo profesional y proyectos especiales.

5. Colaborar en todos los aspectos del Programa de Trabajo Social Escolar. 


\section{Trabajadores(as) Sociales Escolares}

1. Colaborar en los procesos de alianzas con otras organizaciones.

2. Mantenerse actualizados sobre las normativas vigentes que rigen la práctica de TSE y de la profesión en general.

3. Estar disponibles para facilitar la identificación de datos relevantes que aporten a la transformación de las normativas de la práctica de la profesión.

Apoderarse de los datos presentados en las normativas de la profesión y de la práctica de TSE, de manera que se puedan cumplir a cabalidad.

\section{Conclusión}

Los resultados de esta investigación evidencian la necesidad de que se mantengan actualizadas las normativas del Programa de Trabajo Social Escolar, así como de la profesión. También es importante que todos los Trabajadores Sociales Escolares de la conducta se mantengan a la vanguardia de los nuevos conocimientos relacionados con la práctica profesional de trabajo social, de manera que puedan enfrentar los retos que esta plantea. Las respuestas ofrecidas por los encuestados evidencian la importancia que le dan a su profesión los Trabajadores Sociales Escolares. La labor de actualizar las normativas y aspectos relacionados con la profesión de Trabajo Social requiere la integración de los diferentes niveles, sistemas y subsistemas, para que se pueda tener una visión holística e integral de todas las áreas de la profesión: Colegio de Profesionales del Trabajo Social de Puerto Rico, universidades, agencias públicas y privadas. Entre los aspectos que se identificaron como mayor de nivel de importancia se encuentran los relacionados con aspectos éticos de la profesión, los cuales 
se presentan a través de las tres categorías presentadas en el cuestionario: práctica profesional, desarrollo profesional y estructura administrativa y ética.

\section{Referencias}

Asociación Australiana de Trabajadores Sociales. (2003). Estándares de Práctica para Trabajadores Sociales Escolares. Recuperado de www.aasw.asn.au/document/item/814

Asociación Nacional de Trabajadores Sociales. (2012). Estándares para los Servicios que ofrecen los Trabajadores Sociales Escolares de la Asociación Nacional de Trabajadores Sociales (NASW). Recuperado de www.naswdc.org/ practice/standards/naswschoolsocialworkstandards.pdf

Asociación de Trabajadores Sociales de Saskatchewan. (1999). Estándares para Trabajadores Sociales Escolares Registrados.

Burgos, N. (2001). Pioneras de la Profesión de Trabajo Social en Puerto Rico. Puerto Rico: Publicaciones Puertorriqueñas.

Colegio de Profesionales del Trabajo Social. (2011). Código de Ética Profesional: Colegio de Profesionales del Trabajo Social de Puerto Rico.

Colegio de Profesionales del Trabajo Social en Puerto Rico. (2009). Reglamento del Colegio de Profesionales de Trabajo Social en Puerto Rico.

Connecticut. Departamento de Educación. (2014). Estándares para la Práctica de Trabajo Social Escolar. Recuperado de http://www.sde.ct.gov/sde/cwp/view. $\operatorname{asp} ? \mathrm{a}=2663 \& \mathrm{q}=334360$ 
Departamento de Educación de Puerto Rico. (1999). Reglamento de la Carrera Magisterial. Recuperado de www.de.gobierno.pr

Departamento de Educación de Puerto Rico. (2004). Reglamento de Personal Docente de Puerto Rico.

Departamento de Educación de Puerto Rico. (2006). Manual de Procedimientos del Programa de Educación Especial.

Departamento de Educación de Puerto Rico. (2010). Carta Circular 3-2010-2011 Normas para la Organización y Funcionamientos del Programa de Trabajo Social Escolar.

Departamento de Educación de Puerto Rico. (2012). Manual de Procedimientos: Programa de Trabajo Social.

Departamento de Educación de Puerto Rico. (2011). Manual de Protocolos: Guía para el Director Escolar y Personal de Apoyo.

Departamento del Trabajo de Estados Unidos. (2013). Oficina de Estadísticas Laborales: 21-121 Niños, familias y Trabajadores Sociales Escolares. Recuperado de www.bls. gov/oes/current/oes211021.htm

Estado Libre Asociado de Puerto Rico. (2008). Ley Núm. 75 de 30 de mayo de 2008 enmendó el Artículo 6 de la Ley 171 de 11 de mayo de 1940, según enmendada.

Fowler, F. J., Jr. (2002). Survey research methods (3rd ed.). Thousand Oaks, CA: SAGE Publications, Inc.

Linstone, H. \& Turoff, M. (2002). The Delphi Method. Techniques and applications.

Ley de Colegiación de los Trabajadores Sociales, Ley Núm. 171 del 11 de mayo de 1940. Recuperada de www.lexjuris.com 
North Carolina. State Board of Education. North Carolina Professional School Social Work Standards. Recuperada de http://www.ncpublicschools.org/docs/ mah/socialwork/ socialworkstandards.pdf

Red Latinoamericana de Acogimiento Familiar. Relaf. Fondo de las Naciones Unidas para la Infancia, UNICEF. (2010). Guía de estándares para el personal de las entidades públicas y privadas que se ocupan de la protección de derechos de niñas, niños y adolescentes.

Rivera - Colón, I. (2010, agosto). Reunión Profesional de Trabajo Social. Datos del Informe Estadístico de Labor Realizada por el Trabajador Social Escolar 2009-2010.

Verhoeven, P. (2011). Doing research: The How's and Why's of Applied Research. (3rd ed.).Chicago, III. Lyceum Books. 